\title{
Politique
}

\section{Sur le développement international des banques canadiennes}

\section{Marc Chabot}

Numéro 11, hiver 1987

L’État privé

URI : https://id.erudit.org/iderudit/040551ar

DOI : https://doi.org/10.7202/040551ar

Aller au sommaire du numéro

Éditeur(s)

Société québécoise de science politique

ISSN

0711-608X (imprimé)

1918-6584 (numérique)

Découvrir la revue

Citer cette note

Chabot, M. (1987). Sur le développement international des banques canadiennes. Politique, (11), 147-156. https://doi.org/10.7202/040551ar d'utilisation que vous pouvez consulter en ligne.

https://apropos.erudit.org/fr/usagers/politique-dutilisation/ 


\section{Sur le développement international des banques canadiennes}

L'étude de Moreau ${ }^{1}$ se veut la contribution la plus récente consacrée au système bancaire canadien. Essentiellement, l'auteur utilise l'approche marxiste pour appréhender l'internationalisation des banques canadiennes sous une perspective historique. Au terme de son analyse, Moreau conclut à l'insuffisance des théories les plus courantes pour rendre compte de l'internationalisation bancaire canadienne en ce qu'elles font abstraction des facteurs géopolitiques et des circonstances historiques:

«(...) ces théories ${ }^{2}$ souffrent toutes des mêmes défauts. Elles cherchent les racines de l'expansion internationale au niveau de la firme ou de la branche, indépendamment de la dynamique générale de l'accumulation capitaliste. Elles font abstraction des facteurs géopolitiques qui pèsent pourtant très lourd sur le développement international des banques.

«(...) L'articulation concrète du lien entre concentration et internationalisation doit être située en fonction de chaque période historique; il s'agit d'un rapport d'interaction et non de détermination de causalité unilatérale» (p. 149 et p. 151)

Pour Moreau, la théorie de l'impérialisme apparaît comme la plus féconde dans l'analyse du processus de multinationalisation des banques canadiennes (pp. 150-151). Ainsi, le Canada constituerait une puissance impérialiste autonome (p. 84). Toutefois, l'autonomie et le caractère distinct du capital canadien émergerait d'une relation de dépendance successive aux puissances britanniques et américaines (p. 65).

Pour tester la validité de ses hypothèses, l'auteur identifie quatre périodes distinctes dans le processus d'internationalisation des banques canadiennes. Tout d'abord, une période d'hégémonie commerciale et financière britannique qui va jusqu'en 1914. Au cours de cette période, le gouvernement fédéral aurait utilisé les pouvoirs qui lui sont conférés en matière bancaire, commerciale 
et financière pour accélérer le développement de la bourgeoisie canadienne (p. 63). Entre 1914 et 1945, nous assistons à une période de convulsions économiques et politiques profondes accompagnées d'une lutte acharnée pour la domination mondiale. La période d'après guerre est marquée d'un expansion rapide à l'étranger de telle sorte qu'au début des années 80 , «les banques canadiennes sont fortement "surdéveloppées» en comparaison de la taille relative du pays, ainsi que de ses exportations et investissements extérieurs, qui sont pourtant loin d'être négligeables" (p. 110). C'est dans ces conditions que les banques canadiennes entrèrent dans la phase de crise financière des années 80 .

Pour intéressants et révélateurs que puissent être les faits soulevés par l'auteur, l'analyse n'en demeure pas moins insuffisante, croyons-nous, en ce qu'elle néglige d'examiner les rapports des banques canadiennes à l'échelle nationale. En effet, ce n'est que de façon accessoire que l'influence des banques à l'échelle nationale est considérée. L'état des rapports entre les banques et les entreprises industrielles apparaît comme une variable parmi tant d'autres pour rendre compte du processus d'internationalisation bancaire ${ }^{3}$ attendu, toutefois, que «ces rapports ne reflètent pas mécaniquement le niveau de développement économique, mais dépendent fortement des conditions historiques propres à chaque pays» (p. 46). Les observations de Moreau en ce qui concerne les rapports banqueindustrie s'inscrivent donc à l'intérieur d'un ensemble plus vaste d'éléments qui permettent, en quelque sorte, de caractériser le système bancaire canadien.

\section{La caractérisation du système bancaire canadien}

Par delà ses conclusions, l'étude de Moreau présente un intérêt certain en ce qu'elle permet de caractériser le système bancaire canadien. De façon plus particulière, nous avons choisis d'examiner les propos de l'auteur en ce qui concerne les rapports 
avec l'industrie et, notamment, le phénomène des emprunts canadiens en eurodevises de même que ses observations relativement à l'intervention gouvernementale.

\section{Les rapports avec l'industrie}

C'est évidemment en fonction de l'internationalisation bancaire que Moreau examine les rapports banque-industrie. Il observe qu'au cours des années 70, «les banques se développent deux fois plus vite que l'économie canadienne dans son ensemble» et que "les plus grandes entreprises industrielles deviennent des nains en comparaison des banques canadiennes" (p. 114). Le Canada serait le pays où les banques seraient les plus grandes relativement aux industries (et inversement pour les États-Unis). Cette situation serait due à la fois à la force des banques et à la faiblesse des entreprises canadiennes. Et cet écart irait s'élargissant avec le temps (p. 116). Bien que la démonstration à cet effet puisse être discutable ${ }^{4}$, il n'en demeure pas moins que, selon les chiffres de l'auteur, l'actif bancaire total représentait $55 \%$ du PNB canadien en 1970 alors que cette part passait à $104 \%$ en 1982 (p. 114). Moreau exprime en ces termes les conséquences d'une telle disproportion:

La puissance de ses banques constitue un atout de taille pour le capital canadien. En même temps, il place les sociétés emprunteuses dans une situation de dépendance potentielle face aux grandes banques, de qui dépendent leurs possibilités d'acquisition et d'investissement. De plus, les intérêts à verser sur ces emprunts représentent une ponction majeure sur leurs profits, aux taux d'intérêts courants. (...) À mesure qu'elles placent les sociétés industrielles sous leur dépendance, toutefois, les banques tombent elles-mêmes sous la dépendance de la bonne ou de la mauvaise santé de ces industries. En conséquence de cette évolution, les rapports banques/industries deviennent plus intimes y compris sur le plan de leurs activités internationales (p. 131).

L'influence des banques sur l'industrie - et inversement - est sans doute susceptible de s'exercer à différents niveaux. Un aspect intéressant de ces rapports abordés par Moreau a trait aux emprunts canadiens en eurodevises. 


\section{Les emprunts canadiens en eurodevises}

Le marché interbancaire consiste, pour une banque, à déposer ses fonds dans une autre banque plutôt que de les prêter directement à l'utilisateur final. Ainsi uné créance en eurodevise est "une créance sur une banque qui est libellée dans une monnaie différente de la monnaie du pays où la banque est située ${ }^{5}$. Le marché des eurodevises est un marché de gros qui n'est utilisé que par les grandes entreprises, les grandes banques et les gouvernements.

Moreau observe que les banques canadiennes sont passées d'une position de créancier à une position débitrice nette sur le marché interbancaire. En 1969, les dépôts des banques canadiennes dans d'autres banques étaient deux fois plus élevés que ceux en provenance de ces dernières. Au cours de la seule année 1981, le montant net dû par les banques canadiennes est passé de 19,8 milliards $\$$ à 40,2 milliards $\$$ (p. 105). Selon les chiffres compilés par l'auteur, les banques canadiennes viendraient au troisième rang en ce qui concerne les prêts accordés en eurodevises (pour la période 1978-1982) avec $10.2 \%$ du total comparativement à approximativement $35 \%$ pour les États-Unis et $13 \%$ pour la Grande-Bretagne (p. 108). Par ailleurs - et de façon assez paradoxale - , le Canada lui-même se trouvait au deuxième rang parmi les emprunteurs d'eurodevises en 1981 (p. 127).

D'une part, les banques canadiennes comptent donc parmi les principaux prêteurs sur le marché des eurodevises - et cela, en dépit d'une position débitrice nette sur le marché interbancaire - et, d'autre part, le Canada serait un des principaux pays emprunteurs. Poussant l'analyse, Moreau estime que pour 1981, les banques canadiennes auraient fourni $61,4 \%$ des prêts aux compagnies et gouvernements du Canada (p. 128). À partir des prêts publicisés dans la revue Euromoney, l'auteur identifie onze emprunts canadiens en eurodevises. Assez curieusement, nous y retrouvons la Corporation de développement du Canada (la CDC) 
qui a emprunté 2.1 milliards \$U.S. financés en totalité par les banques canadiennes de même que Dome Petroleum (240 millions \$ U.S.) et Massey Ferguson (561 millions $\$$ U.S.) pour lesquelles la part des banques canadiennes étaient toutefois nulles (p. 129).

Nous ne pouvons manquer d'observer que ces emprunts canadiens en eurodevises furent effectués par des entreprises qui sont rattachées, directement ou indirectement, aux principaux cas de sauvetage entrepris par le gouvernement fédéral ${ }^{6}$. Il est malheureux que Moreau n'ait pas accordé plus d'importance aux caractéristiques des entreprises qui empruntent en eurodevises ${ }^{7}$. Il faudrait sans doute s'interroger sur les causes de tels emprunts à l'étranger étant donné les avantages et les inconvénients que peut présenter une telle façon de procéder.

\section{L'intervention gouvernementale}

La caractérisation du système bancaire canadien passe aussi par l'intervention gouvernementale. Pour Moreau, le rôle essentiel des gouvernements est d'agit comme banquier de dernier recours:

«(...) une retraite massive et coordonnée de tous ces dépôts pourrait sérieusement ébranler n'importe quelle banque. Heureusement, ce problème n'est pas critique pour les banques canadiennes. Leurs vastes opérations, alliées au fait qu'elles peuvent compter sur le secours de la Banque du Canada, de même que leur forte position sur le marché international, leur assurent une disponibilité de fonds interbancaires importants dans n'importe quelle éventualité. ${ }^{8}$

C'est en période de crise que se révèle le rôle des États. Ainsi, la création de banques centrales au cours des années 30 s'explique par la nécessité d'aider les banques en difficulté (p. 72). Face à la crise financière mondiale actuelle, les banques canadiennes seraient plutôt vulnérables (pp. 136 ss). Pour les gouvernements, il n'y aurait que trois approches possibles: «subventionner l'emprunteur pour qu'il honore ses engagements, abandonner l'emprunteur à son sort pour combler les pertes de l'intermédiaire financier (la banque), ou encore garantir les dépôts en laissant tomber et l'emprunteur, et la banque» (pp. 138-139). Peut-être 
pourrions nous poser l'hypothèse que le gouvernement fédéral a choisi la première de ces alternatives face à la récente crise financière. L'aide à l'industrie en déclin et, plus particulièrement, le sauvetage d'entreprises pourraient sans doute être examinés sous cette perspective. Par ailleurs, la faillite récente de plusieurs banques et autres institutions financières aux États-Unis pourrait s'expliquer par le choix des autorités gouvernementales de laisser tomber à la fois l'emprunteur et la banque.

\section{Les rapports annuels des banques comme source d'informations}

Un aspect intéressant de l'étude de Moreau est qu'il utilise les rapports annuels des banques pour analyser les opérations internationales des banques canadiennes et, notamment, la diversification géographique des actifs étrangers (pp. 121 ss). Toutefois, il est loin d'être évident que l'information contenue dans ces rapports puisse aussi facilement être comparée. De façon générale, et comme l'indique Moreau (p. 122-123), une certaine confusion résulte du fait qu'une partie des prêts en devises étrangères est effectuée à partir d'établissements situés au Canada. Il faut en effet distinguer entre la part de l'actif total qui est en monnaie étrangère et les activités à l'étranger, c'est-à-dire les opérations effectuées par l'entremise d'établissements situés à l'étranger, peu importe la monnaie utilisée. Sous réserve que certains chiffres présentés par l'auteur (voir tableau XII, p. 122) ne puissent être retracés dans les rapports annuels ${ }^{9}$, les conclusions de l'auteur nous apparaissent tout de même discutables étant donné qu'il considère la totalité des actifs à l'étranger y compris les dépôts auprès d'autres banques. Nous sommes d'avis qu'il y aurait avantage à distinguer l'actif total et les prêts hors banque. À titre indicatif, voici quelle serait la distribution géographique des actifs étrangers de la Banque Royale selon l'une ou l'autre des méthodes: 


\begin{tabular}{lrc}
$\begin{array}{l}\text { (en milliards de } \$ \\
\text { et en } \% \text { du total }\end{array}$ & Actif total & Prêts hors banque \\
\hline Amérique du Nord & $7,9 \$(26)$ & $4,0 \$(27)$ \\
Antilles et Am. latine & $8,0(26)$ & $5,0(33)$ \\
Europe & $12,1 \quad(39)$ & $4,6(31)$ \\
Asie & $2,1 \quad(7)$ & $1,1 \quad(7)$ \\
M.-O. et Afrique & $0,7 \quad(2)$ & $0,3 \quad(2)$ \\
$\quad$ Total & $30,8(100 \%)$ & $15,1 \quad(100 \%)$ \\
\hline
\end{tabular}

Ainsi, la Banque Royale qui vient au deuxième rang pour la proportion de son actif total situé en Amérique latine en détient une proportion encore plus élevée si l'on ne considère que les prêts hors banque. Inversement, elle n'occuperait plus le premier rang pour ses activités en Europe. En résumé, la distinction nous semble pertinente et il est possible d'envisager que certaines des conclusions de l'auteur puissent être infirmées de ce fait.

Cependant, à la défense de Moreau, il faut bien avouer que ce ne sont pas toutes les banques qui distinguent clairement compte tenu de la nature de leurs activités à l'étranger (du moins pour 1981). Et cela est facilement compréhensible dans la mesure où les banques canadiennes ne sont pas assujetties à des normes comptables qui soient identiques. Non seulement les banques ne divulguent pas le même genre d'informations mais les règles suivies lorsqu'elles le font, risquent fort d'être différentes. Il en va ainsi, par exemple, en ce qui concerne la conversion en dollars canadiens d'opérations conclues en devises. Faut-il considérer le taux en vigueur à la date du bilan ou celui au jour où l'opération est survenue? En fait, les règles suivies d'une banque à l'autre étaient à ce point différentes en 1981 que l'Inspecteur général des banques a dû adopter des règlements à cet égard qui sont entrées en vigueur le $1^{\text {er }}$ novembre 1983.

L'utilisation, par Moreau, des rapports annuels des banques comme source d'informations nous révèle l'ampleur des difficultés 
que peut présenter ce procédé. Pour être comparative, l'information présentée se doit d'être redressée au préalable. Cette absence de comparabilité pourrait être examinée de façon globale compte tenu, entre autres, des intérêts particuliers des personnes responsables de la préparation des rapports annuels.

\section{Conclusion}

L'obligation qu'ont les banques de rendre compte de leurs activités renvoie, en quelque sorte, à l'identification des intérêts en présence. Ainsi, pourquoi qualifier de canadiennes les banques établies au Canada? En réponse à cette question, Moreau se contente de faire référence à la thèse de la dénationalisation du capital qui renvoie au transfert plutôt qu'à la simple exportation du capital (p. 62). Il est d'ailleurs révélateur de constater que (sauf erreur), Moreau n'utilise l'expression «bourgeoisie canadienne» qu'en deux occasions (p. 63 et p. 149) et les deux fois pour l'associer au capital britannique et américain. À l'origine toutefois (et même au cours des années 20), les banques canadiennes étaient des banques anglaises (p. 61). En fait, il semble que les banques soient canadiennes dans la mesure seulement où leur existence juridique relève de la loi fédérale sur les banques. Et toute la démonstration de l'auteur repose sur cette simple distinction d'ordre juridique. Nous sommes d'avis qu'il y aurait avantage à examiner plus à fond la formation des banques canadiennes notamment au niveau de la détention de leurs actions et de la gestion de leurs opérations ${ }^{11}$. Et à ce niveau, il serait peut-être utile de considérer, non seulement les banques, mais aussi d'autres institutions financières comme, par exemple, les sociétés de fiducie et les compagnies d'assurance. 
1. F. MOREAU, Le développement international des banques canadiennes, Montréal, Ed. Saint-Martin, 1985, 159 pp.

2. Outre la problématique marxiste, il s'agit d'appliquer aux entreprises bancaires les théories explicatives élaborées pour les multinationales industrielles. Une première approche se fonde sur la minimisation des coûts de transactions sur devises engendrés par le commerce international. Une deuxième école de pensée se situe au niveau de l'analyse de la branche et de la position concurrentielle de la firme qui décide d'investir à l'étranger. La théorie du cycle du produit, celle de la réaction oligopolistique et la thèse des avantages oligopolistiques renvoient à cette seconde approche (voir pp. 16 ss).

3. Les autres variables seraient 1) la dynamique générale de l'accumulation du capital au niveau mondial; 2) le contexte politique global (c'est-à-dire l'évolution des rapports de force politiques entre les grandes puissances capitalistes, entre ces dernières et les peuples dominés, et aussi entre les classes sociales); 3) la position spécifique de chacune des puissances dans le système mondial; et 4) la position spécifique d'une ou plusieurs banques en particulier face à la concurrence (pp. 45-46; voir aussi p. 27).

4. L'auteur compare le chiffre d'affaires des cinq plus grandes firmes industrielles nationales et l'actif des cinq plus grandes banques. Il ignore ainsi toutes les questions relatives à la concentration financière comme, par exemple, l'importance relative des compagnies privées (celles dont les titres ne sont pas transigés dans le public) ou encore la participation des sociétés d'État.

5. Lindert et Kinkleberger, Économie internationale, $7^{\text {ième }}$ éd., Paris, Economica, 1983 , p. 496.

6. Un peu plus de $48 \%$ des actions de la CDC sont contrôlées par le gouvernement fédéral par l'entremise de la Corporation des investissements du Canada (la CDIC). Cette dernière contrôle également les activités des entreprises suivantes : Canadair, De Havilland, Eldorado Nuclear, Teleglobe, Massey Ferguson et trois pêcheries des provinces de l'Atlantique.

7. En fait, Moreau s'intéresse plus à la distribution géographique des prêts consentis par les banques canadiennes à des étrangers (toujours sur le marché des eurodevises). Ces derniers comptent pour $61.4 \%$ des prêts internationaux consentis par les banques canadiennes (à ne pas confondre avec la part des banques canadiennes dans les emprunts canadiens en eurodevises pour 1981 qui est identique).

8. Kitchell, "Les banques canadiennes sur les marchés internationaux», Le banquier et Revue IBC, no. 3, 1981, p. 43 tel que cité p. 137 . Notons au passage que Moreau n'aborde l'intervention gouvernementale que sous ce seul aspect.

9. Nous n'avons pas examiné de façon systématique tous les chiffres présentés au tableau XII. Sous cette réserve, mentionnons que, la répartition géographique des actifs étrangers de la Banque Royale ne correspond pas aux pourcentages indiqués dans le rapport annuel. Ainsi le pourcentage des prêts effectués en Amérique du Nord serait de $29 \%$ et non de $26 \%$ (voir p. 33 du rapport annuel). Dans ce dernier cas, il semblerait que l'auteur ait utilisé les pourcentages de 1980 plutôt que ceux de 1981. Ajoutons que le total des actifs étrangers de la Banque de Montréal seraient de 25,4 milliards \% (et non de 22,4) dont 12,9 milliards $\$$ (et non 8,8) en Amérique du Nord (voir p. 61 du Rapport annuel). Nous n'avons pas examiné les rapports annuels de la Banque de Commerce Canadienne Impériale et ceux de la Toronto-Dominion.

10. Nous avons utilisé les chiffres de Moreau en ce qui concerne l'actif total en monnaie étrangère et cela, en dépit de l'écart mentionné précédemment (voir note 9). Selon le rapport annuel de la Banque Royale, la répartition géographique serait plutôt la 
suivante: Amérique du Nord $29 \%$, Antilles et Amérique Latine $22 \%$, Europe $39 \%$, Asie $8 \%$ et finalement M.-O. et Afrique $2 \%$.

11. Il est à noter que la législation bancaire actuelle comporte des restrictions quant au nombre d'actions qui peuvent être détenues par des non-résidents (voir, par exemple, Loi sur les banques, S.C. 1980-81, art. 110 (1) qui établit une limite de $25 \%$ au total pour chaque catégorie d'actions). Sans doute serait-il intéressant de savoir à quelle époque remonte l'établissement de telles restrictions.

\section{Marc Chabot \\ Université du Québec à Montréal}

